\title{
Gas identification and estimation of its concentration in a tube using hyperspectral thermography approach
}

\author{
by R. Olbrycht*, M. Kałuża*, W. Wittchen**, M. Borecki**, B. Więcek*, G. De Mey*** \\ * Lodz Univ. of Technology, 90-924, Wolczanska Str., Lodz, Poland, robert.olbrycht@p.lodz.pl \\ ** Institute for Ferrous Metallurgy, K. Miarki St. 12-14, 44-100 Gliwice, Poland, wwittchen@imz.pl \\ *** Univ. of Ghent, Department of Electronics and Information Systems, Sint Pietersnieuwstraat 41, \\ 9000 Ghent, Belgium, demey@elis.ugent.be
}

\begin{abstract}
The aim of this paper is to demonstrate the application of reflective diffraction grating and MWIR thermal camera for identification of gas and estimation of its concentration. For this purpose a special rig was created with airtight tube that was filled with different mixtures of carbon monoxide, carbon dioxide and nitrogen. Thanks to infrared windows at both sides of this tube, the radiation from blackbody could pass through it and diffract at the reflective diffraction grating to finally reach the camera. After spectral calibration this rig may be used to identify gas in a tube, because different gases have different absorption bands. Measuring signal in the absorption band one can estimate the concentration of gas in a tube.
\end{abstract}

\section{Introduction}

Energy of infrared radiation is sufficient to cause vibrations or rotations of molecules of some gases. Therefore infrared absorption in gases is dependent on its molecular structure and the existence or lack of dipole moment. In case of permanent dipole moment, it is possible to cause rotations. On the other hand, gases with diatomic molecules as $\mathrm{O}_{2}$, $\mathrm{H}_{2}$ or $\mathrm{N}_{2}$ (with zero dipole moment) are transparent to infrared radiation [1].

For linear and nonlinear molecules the number of vibrational modes is different, equal to respectively $3 \mathrm{~N}-5$ and $3 \mathrm{~N}-6$ (where $\mathrm{N}$ is the number of atoms). Hence, for example, a $\mathrm{CO}_{2}$ molecule containing 3 atoms will have $3 \times 3-5=4$ fundamental vibrational modes. The four vibrational modes of $\mathrm{CO}_{2}$ molecule are presented in fig. 1. A carbon dioxide molecule can be stretched (symmetrically (a) or asymmetrically (a)) or bent in two different planes ((c) or (d)) [1].

In the (a) vibrational mode both carbon dioxide $\mathrm{C}=\mathrm{O}$ bonds are stretched and contracted symmetrically, whereas in the (b) mode they are stretched and contracted asymmetrically. The asymmetric (b) stretching is infrared active, because it is leading to dipole moment changes. This particular vibrational mode is associated with $4.26 \mu \mathrm{m}$ radiation wavelength and is the strongest $\mathrm{CO}_{2} \mathrm{IR}$ absorption band. The symmetric (a) vibration mode is not infrared active, as it is not leading to dipole moment changes. The two equal-energy bending vibrational modes in carbon dioxide ((c) and (d) in fig. 1) are identical, except that the first one is occurring in the paper plane and the second one is out of the plane [1].

a)

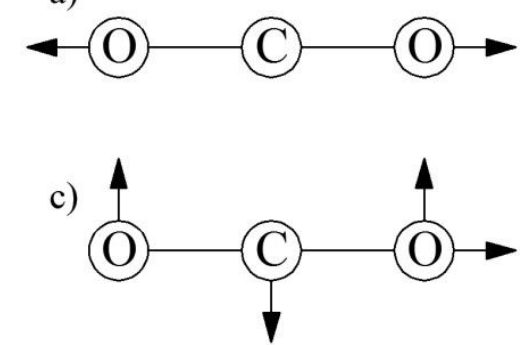

b)

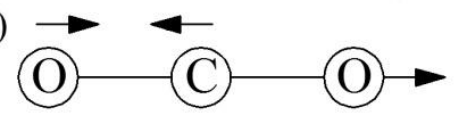

d)

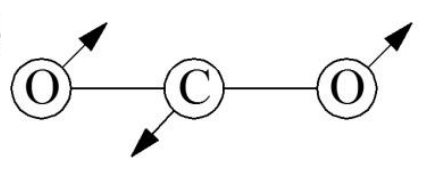

Fig. 1. $\mathrm{CO}_{2}$ molecule vibrational modes: only b-c-d are leading to infrared radiation absorption [1]

Because infrared radiation is absorbed in narrow spectral bands by some gases, hyperspectral imaging may be used to measure the wavelengths being absorbed by an unknown gas. Its identification requires the knowledge of theoretical absorption bands for different gases to correlate it with measurement results. This way the authors managed to identify carbon monoxide and carbon dioxide in a measurement tube - the experimental rig is described in the following paragraph. 


\subsection{1/qirt.2016.095}

\section{Measurement rig}

The authors elaborated the test rig shown in fig. 1 where reflective diffraction grating is applied to provide hyperspectral imaging with the thermal camera. The idea of such a system and formulas for angle calculation are presented in [1]. The radiation diffracted on the grating comes from the blackbody model and passes through the slit and the measurement tube with unknown gas mixture. This is possible thanks to ZnSe windows (transparent for infrared radiation) at both tube ends. The camera acquires the image of the vertical slit that is diffracted horizontally according to the wavelength. Because the blackbody has continuous emission spectra, the whole image should be bright. If the gas is present in the tube, and it absorbs infrared radiation at certain wavelengths, one will see it as dark absorption vertical lines (or bands) [2].
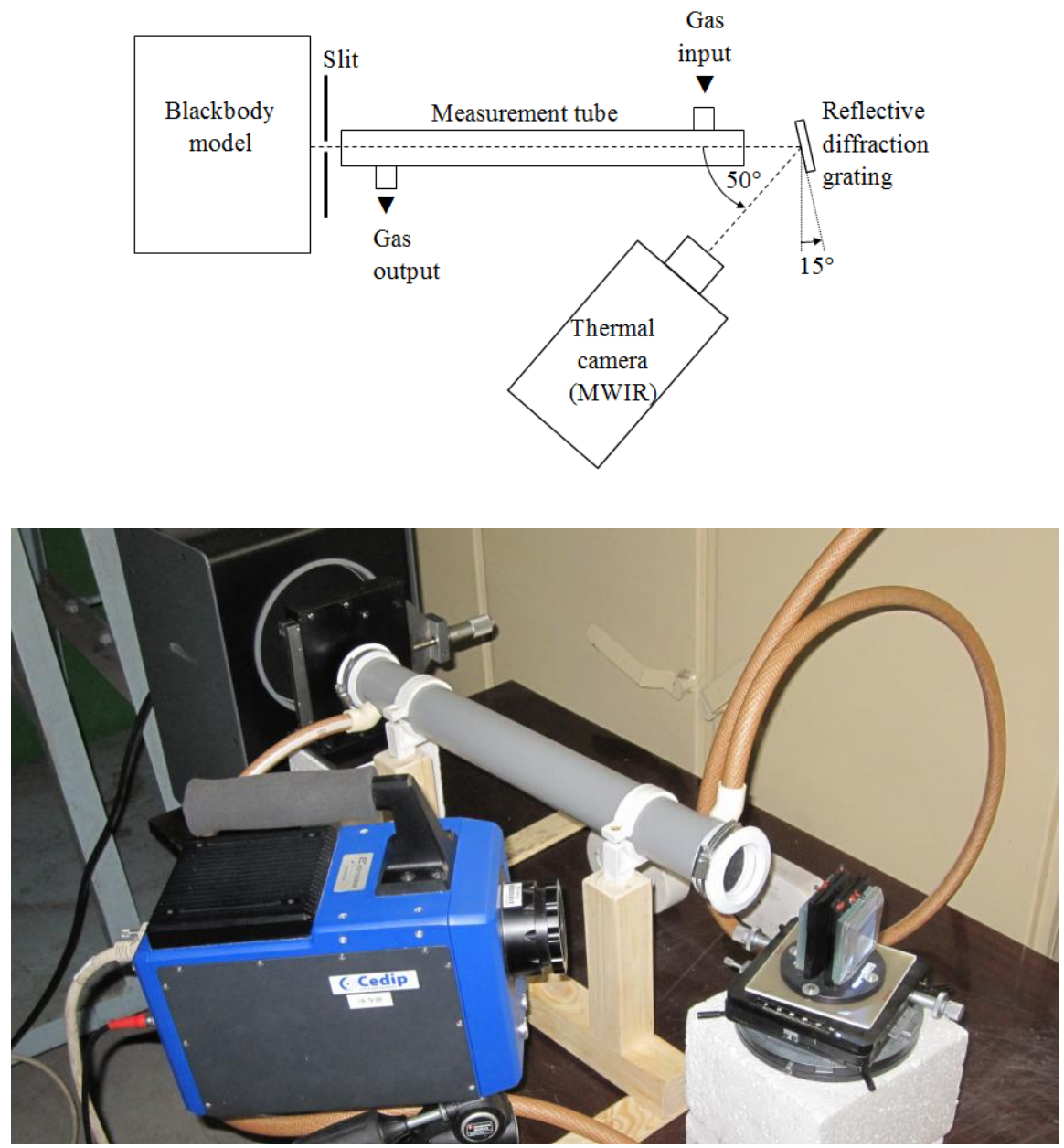

Fig. 2. The measurement test rig

\subsection{Spectral calibration}

There are two calibrations required in such a system. The first one is a spectral calibration, which is necessary when one wants to know the wavelengths corresponding to different image horizontal positions. This calibration may be carried out by filling the tube with absorption medium exhibiting known spectral transmission. There are reference databases with this transmission data, e.g. [3]. In case of this paper the spectral calibration was performed with carbon monoxide and carbon dioxide - the recorded transmission spectrum of mixture containing $18.8 \% \mathrm{CO}, 19 \% \mathrm{CO}_{2}$ and $62.2 \% \mathrm{~N}$ is shown in fig. 2a. For the purpose of clear visualization, however, it is worth subtracting this spectrum from the one shown in fig. $2 b$, which is recorded for pure nitrogen in the tube. In result, the differential spectrum is obtained, as shown in fig. 2c. There are clearly visible two absorption bands, what is also demonstrated in fig. $2 \mathrm{~d}$, where vertically averaged horizontal cross-section of fig. $2 \mathrm{c}$ is plotted with the red line. Knowing theoretical absorption bands of these gases, one can introduce wavelength scale, as shown in fig. $2 \mathrm{~d}$, so that theoretical and practical curves match. For comparison, in fig. $2 \mathrm{~d}$ there is also a spectrum shown with blue color for mixture of $19.8 \% \mathrm{CO}$ and $80.2 \% \mathrm{~N}$. 

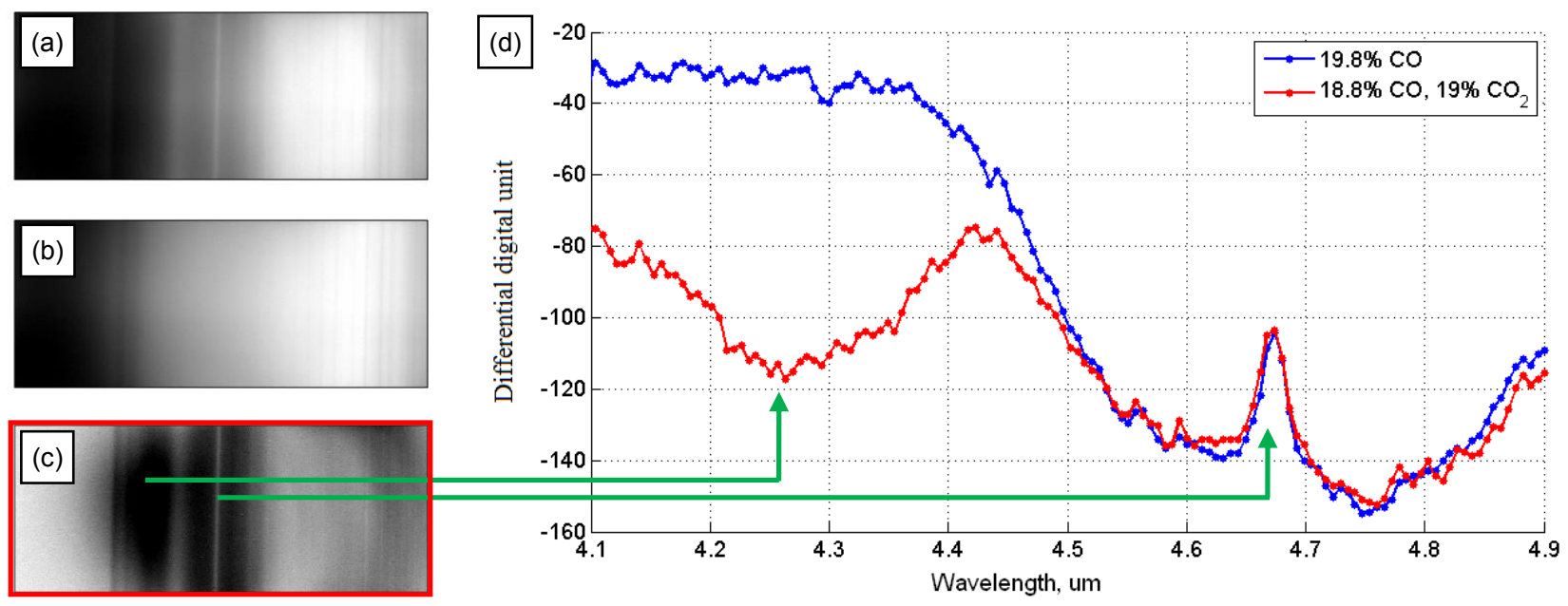

Fig. 3. Transmission spectra of (a) mixture containing $18.8 \% \mathrm{CO}, 19 \% \mathrm{CO}_{2}$ and $62.2 \% \mathrm{~N}$, (b) pure nitrogen, (c) difference (a) - (b), (d) vertically averaged cross-section of (c)

\subsection{Calibration for gas concentration}

The second calibration required in the described system enables gas concentration measurement. Having gas input and output mounted at both ends of the tube it is possible to fill it with certain gas mixture, carry on infrared measurement and later on direct this gas mixture to reference measurement apparatus (not shown in fig. 1). This way the differential infrared measurement (expressed in differential digital units) may be correlated with reference apparatus measurement to provide infrared system calibration for gas concentration measurement.

During the calibration process there were nine differential spectra recorded for chosen concentrations of carbon monoxide mixed with nitrogen. Examples of these are shown in fig. 4. Although it may seem almost identical, the difference is revealed by looking at the scale (expressed in differential digital units) - with higher concentration the values are lower. This is because the increase of gas concentration causes the infrared transmission factor to decrease, according to the Beer's law [4].

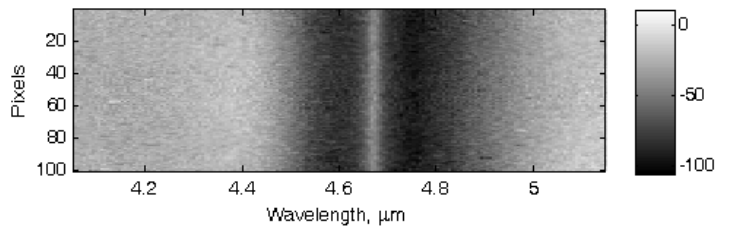

(a)

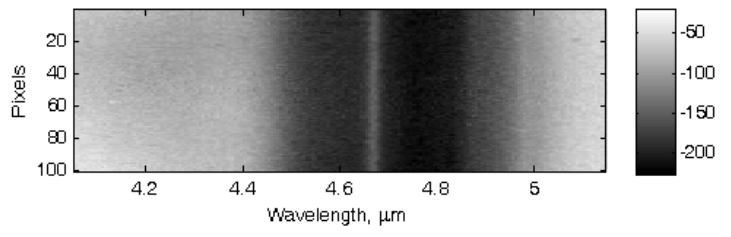

(c)

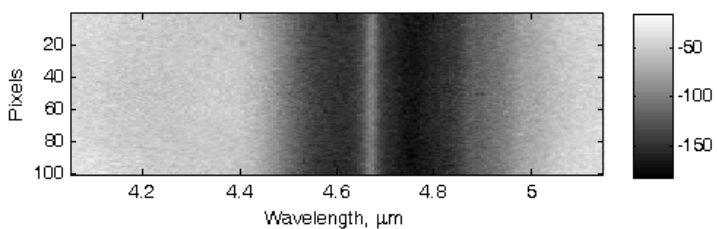

(b)

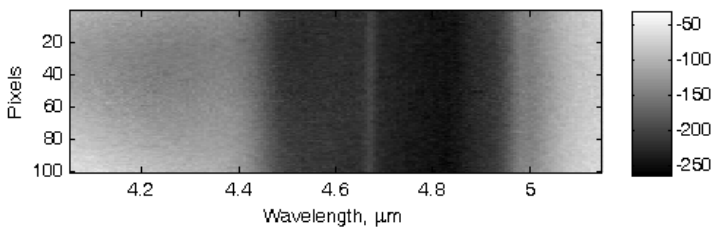

(d)

Fig. 4. Exemplary carbon monoxide transmission spectra mixtures of nitrogen with (a) $3.4 \%$, (b) $10.4 \%$, (c) $30 \%$, (d) $75 \%$ carbon monoxide content

Having recorded above mentioned nine spectra, it was possible to plot vertically averaged horizontal crosssection for each of it - fig. 5. As expected, increasing concentration of carbon monoxide caused higher absorbance. Due to relatively low spectral resolution the peak at $4.67 \mu \mathrm{m}$ is not going to zero level, as it could be expected basing on theoretical transmission data for this gas [3]. So the differential values at these peaks for all nine spectra were measured and plotted in fig. 6 . 


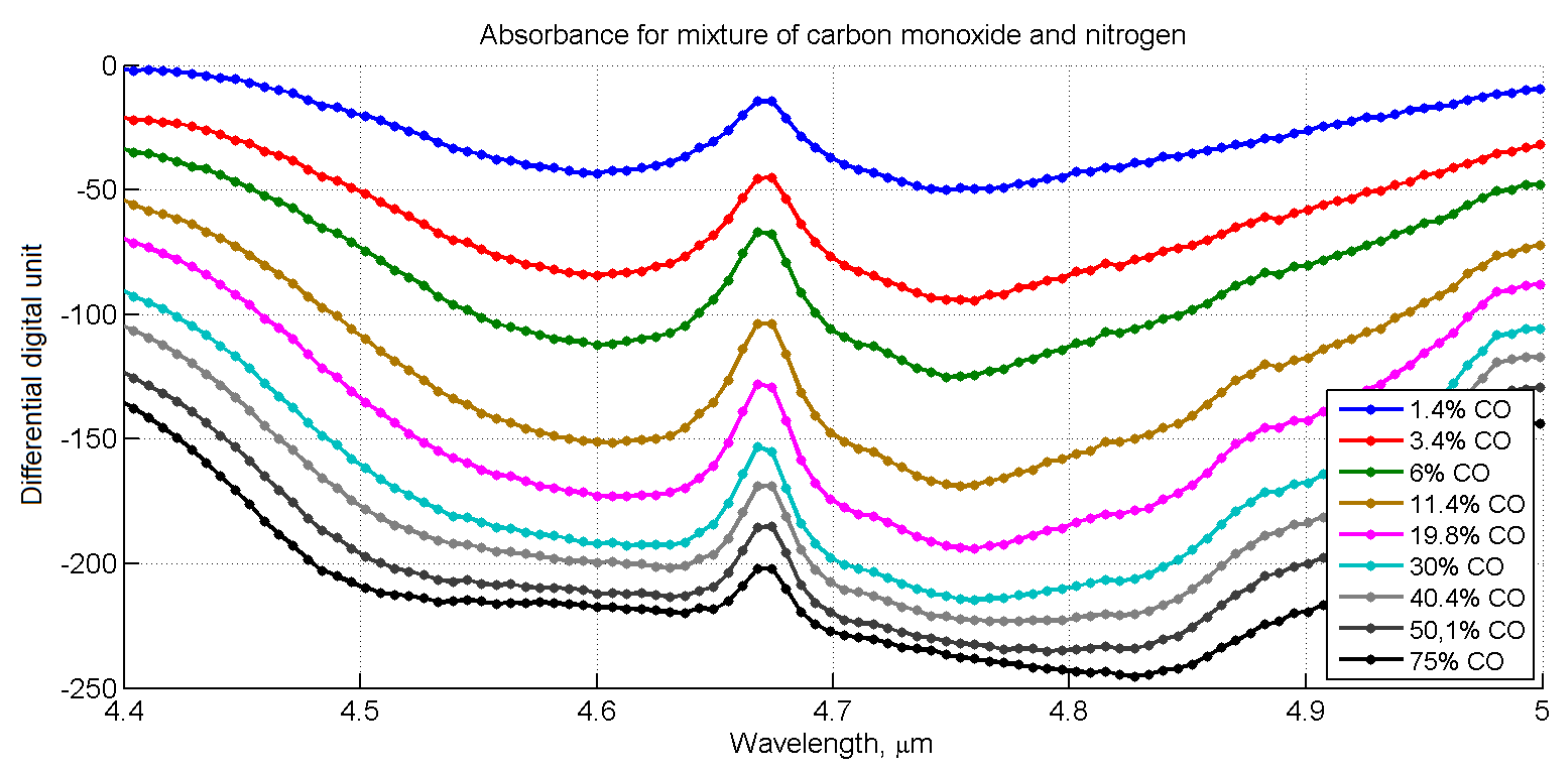

Fig. 5. Transmission spectra for mixtures of nitrogen with given carbon monoxide content

To calculate the calibration curve it is necessary to apply curve fitting. The Beer-Lambert law (also known as Bouguer law) given by (1) seems to be the most appropriate model because it describes the transmission factor versus path length and absorption coefficient. Considering the measurement values, however, it is necessary to add another offset coefficient to this equation, as given by (2), to enable curve fitting with measured differential data.

$$
\begin{gathered}
I=I_{0} \cdot e^{-a \cdot L} \\
I=I_{0} \cdot e^{-a \cdot L}-b
\end{gathered}
$$

where:

$I \quad$ - intensity of radiation after passing the tube

$I_{0}$ - initial intensity of radiation before entering the tube

a - absorption coefficient of the gas inside tube, which is linearly dependent on its concentration

$L$ - length of the tube

$b-$ offset value required for curve fitting with measured differential data

During the curve fitting process, the best matching values of $I_{0}=190.14, L=0.055$ and $b=196.32$ were found, enabling us to plot the calibration curve in fig. 6 using equation (2). As it may be observed, this calibration curve is very close to real measurement. It is confirmed by the correlation coefficient value equal to 0.995 and standard error of 8.026 . Using the found values, one may estimate the concentration of gas in a tube by applying the equation (3), which is derived from (2).

$$
a=-\frac{\ln \left(\frac{I+b}{I_{0}}\right)}{L}
$$

What is important, it is possible to calculate calibration curves for different lengths of the tube, using equation (2). Let us check this dependence for tube lengths equal to $25 \mathrm{~cm}, 50 \mathrm{~cm}$ (original size), $100 \mathrm{~cm}$ and $200 \mathrm{~cm}-$ the results are shown in fig. 7 . 


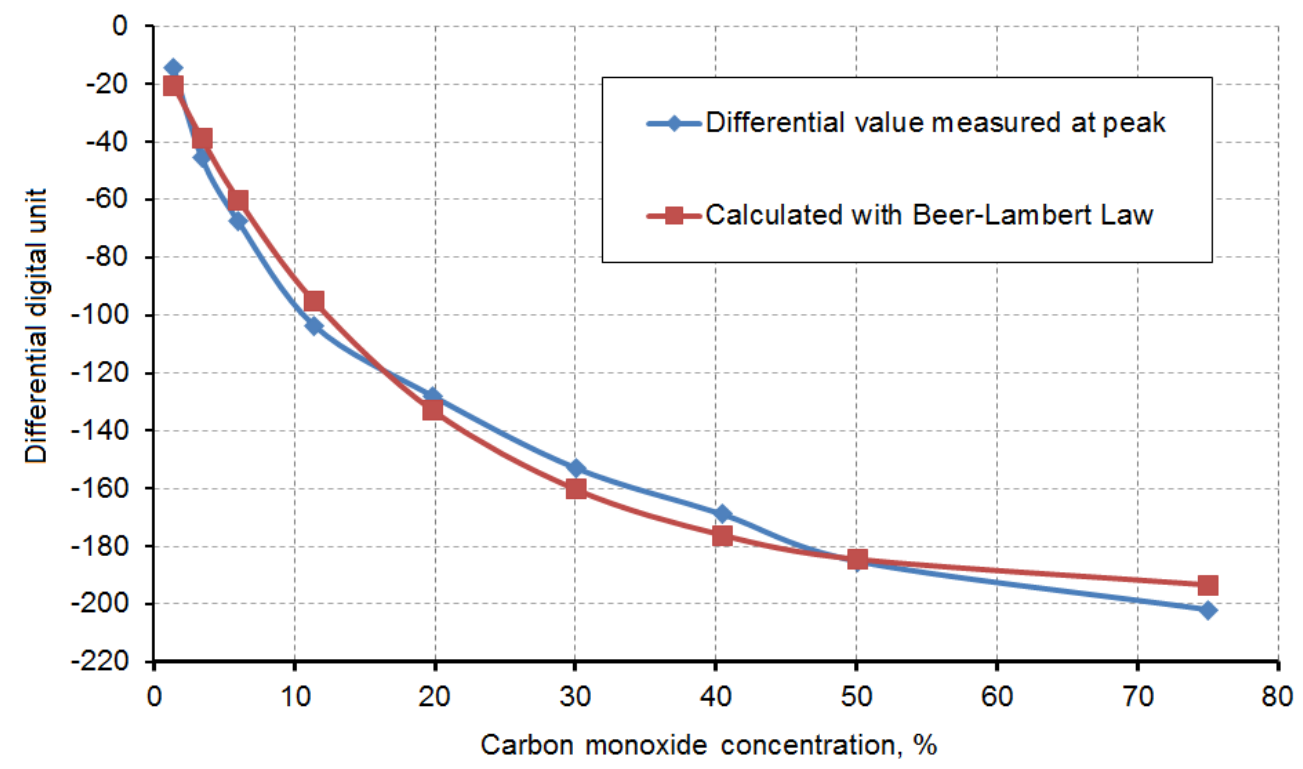

Fig. 6. Theoretical and measured calibration curves for carbon monoxide concentration measurement

Basing on fig. 7 it may be observed that the sensitivity of a system and its measurement range are interrelated. Increasing the tube length causes higher sensitivity but saturation will occur at lower gas concentration thus limiting the measurement range. In case of the particular system discussed in this paper, the tube length was equal to $50 \mathrm{~cm}$. It seems the best compromise between the sensitivity and measurement range. However when one needs to measure high concentrations (above $50 \% \mathrm{CO}$ ) the tube length may be twice (or even more) shorter to provide adequate sensitivity. On the other hand, when high sensitivity is required to measure low CO concentrations (below 10\%) the tube should be about four times longer.

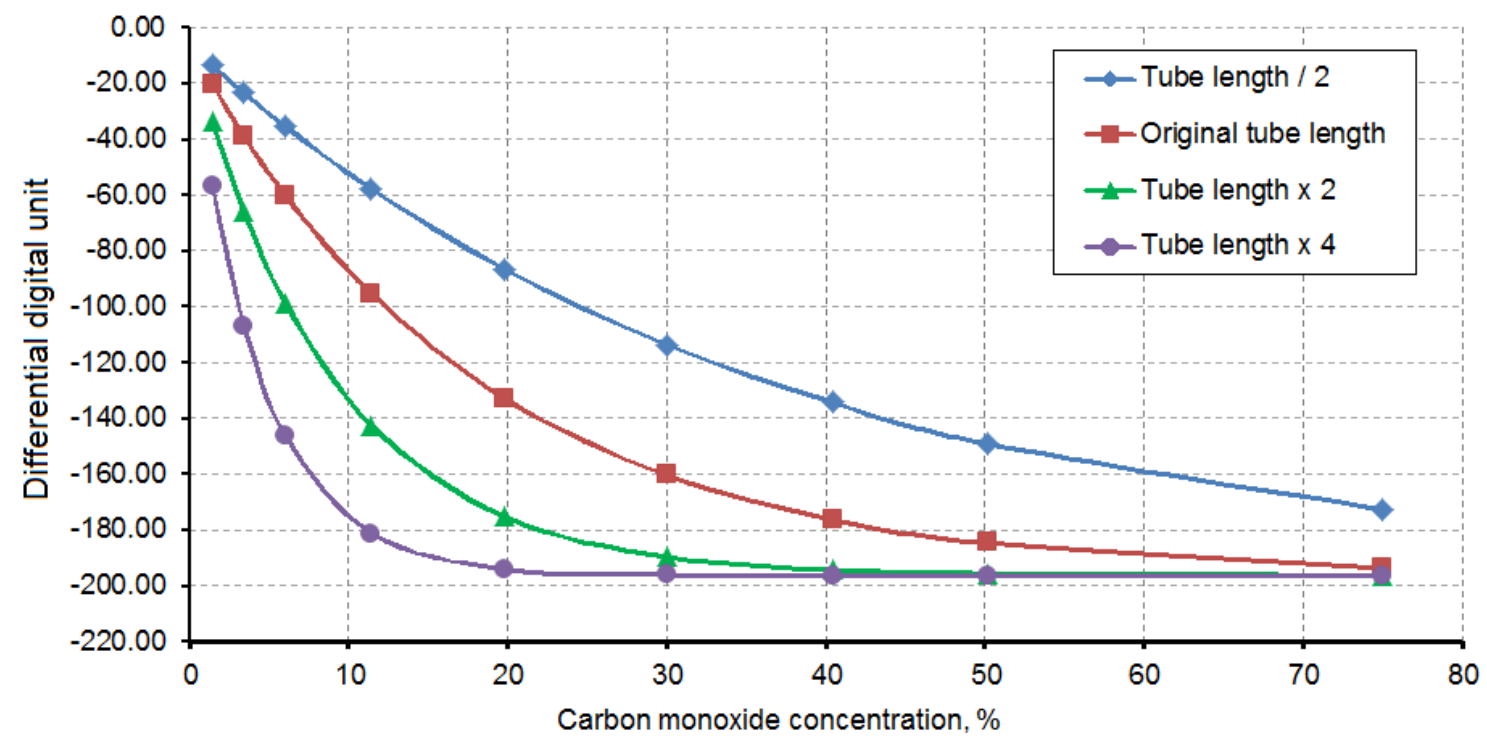

Fig. 7. Theoretical calibration curves for carbon monoxide concentration measurement for different tube lengths 


\subsection{1/qirt.2016.095}

\section{Conclusions}

Broadband thermal camera may be used for hyperspectral measurements thanks to a diffraction grating. There is also a slit and radiation source necessary. The authors demonstrated the application of such a system to identification of gases in a tube and measuring its concentrations. It requires two initial calibrations. The first one is for wavelength versus horizontal pixel number and the second one is for gas concentration versus measured differential signal. In case of this paper the first calibration was done with carbon monoxide and dioxide while the second one only for carbon monoxide. Measurements results are in good agreement with Beer's law. The sensitivity of this system may be controlled by changing the gas tube length. There is also another important advantage of the proposed solution - it may work in real time without any need for scanning, what is often found in many hyperspectral solutions.

If the gases in a tube are known and there is no need to identify it, this system may be simplified to nondispersive apparatus based on two (or more, depending on number of gases) infrared detectors (without thermal camera) - such solutions are described in literature, e.g. [5]. More information about optical gas sensing may be found in e.g. [6]

\section{REFERENCES}

[1] R. Olbrycht, B. Więcek, M. Kałuża, Spectral infrared analysis in thermal cameras with diffraction gratings, QIRT 2014 Conference, Bordeaux

[2] C. Palmer, E. Loewen, Diffraction Grating Handbook, $6^{\text {th }}$ edition, Newport Corporation, 2005

[3] P.J. Linstrom and W.G. Mallard, Eds., NIST Chemistry WebBook, NIST Standard Reference Database Number 69, National Institute of Standards and Technology, Gaithersburg MD, 20899, http://webbook.nist.gov, (retrieved May 10, 2016).

[4] D. F. Swinehart, The Beer-Lambert Law, Journal of Chemical Education 196239 (7), 333, DOI: $10.1021 /$ ed039p333

[5] J. Hodgkinson, R. Smith, W. Ho, J. Saffell, R. Tatam, Non-dispersive infra-red (NDIR) measurement of carbon dioxide at $4.2 \mu \mathrm{m}$ in a compact and optically efficient sensor, Sensors and Actuators B: Chemical 186, 580-588

[6] J. Hodgkinson, R. Tatam, Optical gas sensing: a review, Measurement Science and Technology 24 (1), 012004 